\title{
MBSE-Ansatz für eine Vernetzte \\ Stoffstrommodellierung zur Verbesserung der Partnersuche in der Kreislaufwirtschaft
}

Franz Wieck, Philipp Kronenberg, Manuel Löwer

Globale Krisen, Rohstoffengpässe oder Preisschwankungen stellen die globalisierten Lieferkettensysteme der westlichen Produzenten vor große Herausforderungen. Das Konzept der Kreislaufwirtschaft ist eine Möglichkeit, sich angesichts dieser Herausforderungen robuster zu positionieren, um weniger abhängig von äußeren Einflüssen zu sein. Die größte Hürde bei dem Aufbau von Netzwerken für Kreislaufwirtschaftsprozesse ist die Identifikation von potentiellen Partnern auf Basis von Stoffstrommodellen. Ein erfolgreiches Mapping ist von vielen Faktoren abhängig.

Aktuell existiert kein Ansatz, um die Stoffströme so zu modellieren, dass die Darstellung und Berechnung für eine Partnersuche in der Kreislaufwirtschaft geeignet ist. Um der Komplexität, sowohl der Stoffstrommodellierung, als auch der Partnersuche gerecht zu werden, wird hier ein MBSE-Ansatz gewählt - ein überlegener Modellierungsansatz im Detaillierungsgrad und der Adaptionsfähigkeit

Das Modell verknüpft die 3 Hauptelemente der Wertstromanalyse (Produktionsprozess, Materialfluss und Geschäftsprozess) und ermöglicht dadurch neuartige Kennzahlen zu ermitteln. Diese Kennzahlen und die detaillierte Modellierung schaffen eine verbesserte Informationslage, auf Basis derer die Effektivität der Partnersuche in der Kreislaufwirtschaft signifikant gesteigert wird. Nach einer theoretischen Herleitung der Modellierungslogik und der Erweiterung des bestehenden Ansatzes der nachhaltigen Wertstromanalyse (engl. Sustainable Value Stream Mapping - SUs-VSM) wird anhand eines Beispiels das MBSE-Modell implementiert und validiert.

Dieser MBSE-Ansatz besitzt das Potential, die Beschreibung industrieller Produktions- und Herstellungsprozesse erheblich zu verfeinern und dadurch Analysen und Berechnungen zu optimieren, was zu einer besseren Vernetzung der Industrie führt. Die dadurch identifizierten industriellen Symbiosen fördern die Kreislaufwirtschaft maßgeblich und helfen Ressourcen nachhaltiger zu nutzen.

Keywords: MBSE, Stoffstrommodellierung, Kreislaufwirtschaft, Wertstromanalyse, Sus-VSM; Nachhaltigkeit 


\section{Einleitung}

Im August 2020 hat die Menschheit alle natürlichen Ressourcen aufgebraucht, die die Erde innerhalb eines Jahres wiederherstellen kann. Dieser Tag wird als Earth Overshoot Day (Erdüberlastungstag) bezeichnet und bedeutet für die Erde, dass nach diesem Tag die Weltbevölkerung mit einem Ressourcendefizit agiert (Global Footprint Network 2020). Um den aktuellen Verbrauch an Ressourcen zu decken wären 1,5 Erden notwendig. Die Notwendigkeit bestehende Ressourcen zu schützen und in Kreisläufen so lange wie möglich zu nutzen, bleibt demnach eine globale Herausforderung (Umweltbundesamt 2019).

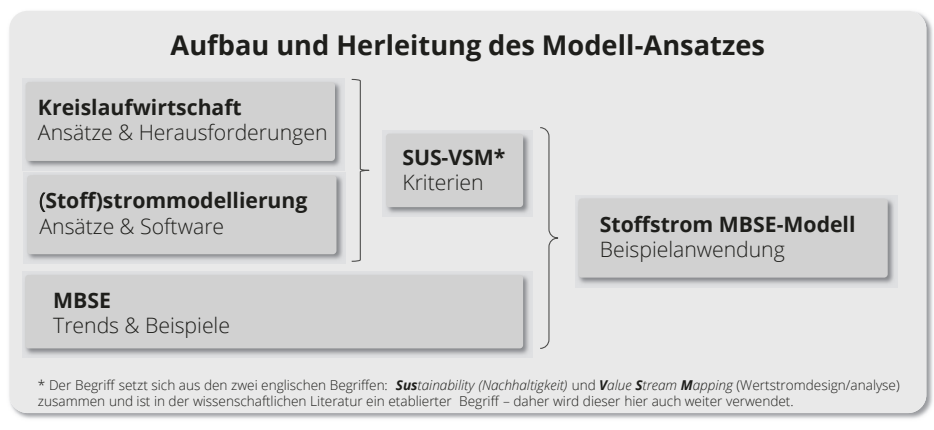

Abbildung 1: Theoretischer Aufbau und Herleitung des MBSE-Modells

Nachhaltigkeit und das Bewusstsein darüber ist schon seit längerem bei Firmen und Kunden existent. Marketingbotschaften werben immer stärker mit „nachhaltigeren Produkten“ oder "grünen Lösungen“. Die Frage ist nur, wie kann in komplexen internationalen Lieferketten die Nachhaltigkeit berechnet und transparent für Kunden und Hersteller aufgezeigt werden. Diese Verantwortung gegenüber Zuliefererstrukturen, auch über die Landesgrenzen der Europäischen Union hinaus, hat das aktuelle Lieferkettengesetz im Fokus. Es verpflichtet Unternehmen ihrer Verantwortung gegenüber ihren Zulieferern in Bezug auf Umweltschädigungen, Arbeitsbedingungen und weiteren Aspekten wahrzunehmen. In Zukunft stehen Unternehmen vor der Herausforderung diese komplexen Netzwerke abzubilden und zu analysieren. Der hier vorgestellte MBSE-Ansatz bietet die Möglichkeit komplexe Netzwerke aufzunehmen und zu berechnen. Anhand eines Praxisbeispiels wird die Möglichkeit demonstriert und erprobt.

Der Artikel ist wie folgt aufgebaut (Abbildung 1). Zunächst wird eine kurze Einführung in Model Based Systems Engineering gegeben. Dann wird das Konzept der Kreislauf- 
wirtschaft mit besonderem Fokus auf Geschäftsmodellentwicklung und aktuellen Barrieren erörtert. Als zentraler Bestandteil wird dann die Wertstromanalyse in Bezug auf die Nachhaltigkeit diskutiert. Anschließend erfolgt die Vorstellung eines Rahmenwerks, welches die Nachhaltigkeit berücksichtigt und die Stoffstrommodellierung detailliert abbildet. Dieses Rahmenwerk wird dann anhand eines Beispiels, welches in SysML implementiert wurde, im Detail analysiert. Der Beitrag schließt mit der Diskussion über die Verbesserung und Erweiterung des entwickelten Models.

\section{Theoretischer Hintergrund}

\section{Model Based Systems Engineering}

Modellbasiertes Systems Engineering (Model-Based-Systems-Engineering - MBSE) ist eine Methodik, in der Informationen über ein System nicht mehr ausschließlich auf Dokumenten basieren, sondern auf Modellen. In der Regel werden diese Modelle auf Basis der UML- oder SysML-Spezifikationen erstellt. Obwohl sich MBSE in Wissenschaft und Forschung immer größerer Beliebtheit erfreut und die Zahlen der Veröffentlichungen stetig steigen, sind viele Fragen und der anwendungsbezogene Nutzen von MBSE aktuell immer noch ungeklärt. Ein großer Teil der aktuellen Veröffentlichungen thematisiert MBSE im (Produkt)Entwicklungskontext. Dabei werden die Vorteile einer modellbasierten Entwicklung in der Analyse, Simulation und Verifikation (mechatronischer) Systeme betrachtetet (Barbedienne et al. 2019; Bjorkman et al. 2013; Mitchell 2014) und die Unterstützung bei Projekt- bzw. Entwicklungsmanagementaufgaben (Andersson et al. 2010; Bott und Mesmer 2020; Madni 2015). Neben diesem Fokus existiert ein weiterer, der den Herstellungs- und Produktionskontext unter der Berücksichtigung von MBSE-Ansätzen betrachtet. Digitale Zwillinge physischer Elemente (Delbruegger und Rossmann 2019), die Kombination und Wechselwirkung zwischen kundenspezifischen Bestellungen und firmeninternen Produktionsprozessen zur Herstellung verschiedener Produktvarianten (Benavent Nacher et al. 2020; Wu et al. 2013), eine adaptive Berechnung von Maschinenkapazitäten am Beispiel von cloudbasierten 3D-Druckern (Luo et al. 2020), Beschreibungen eines verlustfreien Informationsaustausches zwischen Produkt- und Prozessdaten mithilfe von MBSE (Tchoffa et al. 2019) und die Modellierung von Bewegungen, entweder im Kontext von Flughäfen (Keskin und Salman 2020), Warenhäuser (Sprock et al. 2017) oder anderen Infrastrukturen (Poller 2020) sind Beispiele für derzeitige Anwendungsfelder. So existieren aktuell viele Teillösungen, aber Modellierungs-Methodiken und gesamtheitliche Ansätze für gesamte Industriezweige oder Produkte fehlen - so auch ein MBSE-Ansatz für die das immer wichtiger werdende Thema Kreislaufwirtschaft. 


\section{Kreislaufwirtschaft - Potentiale und Hemmnisse}

Das Ziel einer Kreislaufwirtschaft ist es, Ressourcen so lange wie möglich zu nutzen. In der aktuellen Wissenschaft existieren viele Definitionen und Maßnahmen für die Kreislaufwirtschaft. Eine sehr ausführliche Literaturrecherche über die aktuellen Definitionen geben Kirchherr und Su (Kirchherr et al. 2017; Su et al. 2013). Für das in diesem Aufsatz angestrebte Ziel mittels Stoffstrommodellierung die Herausforderungen der Kreislaufwirtschaft zu verbessern, um somit nachhaltigerer Geschäftsprozesse zu ermöglichen, werden zwei bestehende Definitionen von Kreislaufwirtschaft kombiniert. Die Definition von Yuan stellt bei der Definition den Materialfluss in den Mittelpunkt und fordert für eine Kreislaufwirtschaft, dass ein geschlossener Materialfluss angestrebt werden muss (Yuan et al. 2006). Diese Beschreibung gilt es um die Geschäftsprozessperspektive von Bocken (Bocken et al. 2016) zu erweitern. Daraus folgt: Kreislaufwirtschaft zeichnet sich durch Geschäftsmodellstrategien aus, die Stoffströme schließen.

Der aktuelle wissenschaftliche Diskurs für die Kreislaufwirtschaft wird vor allem durch zwei Hauptthemen bestimmt: Geschäftsmodell-Entwicklung, Barrieren und Hemmnisse. Diese werden im Folgenden näher erörtert, um die Herausforderungen und die daraus resultierenden Lösungsvorschläge mithilfe von MBSE zu beschreiben.

\section{Geschäftsmodell-Entwicklung}

Pieroni hat eine umfassende systematische Literaturrecherche zu innovativen Geschäftsmodellen im Kontext der Kreislaufwirtschaft durchgeführt (Pieroni et al. 2019). Dabei wird eine Kategorisierung verwendet, welche die dynamischen Eigenschaften von Unternehmen, auf externe Randbedingungen zu reagieren, in ein 3 Phasen-Modell aufteilt: Erkennen, Ergreifen und Umsetzen (Teece 2007). Die Autoren dieser Übersicht kommen zu dem Ergebnis, dass die letzte Phase, die Umsetzungsphase, stark unterrepräsentiert ist und begründen das durch folgende Argumentationskette: Es fehlt an Methoden, um die Ideen und Konzepte systematisch zu erproben. Ohne diese Erprobungsphase ist das Risiko einer Implementierung zu hoch und findet somit nicht statt. Weiterhin kommen die Autoren zu 3 Ergebnissen bei Problemen von Geschäftsmodellen: das fehlende Prozessverständnis, die Abweichung von klassischen Geschäftsmodell-Strukturen und die fehlende Einbeziehung des menschlichen Verhaltens in die Vorgehensweisen. Der hier vorgestellte Ansatz fördert das Prozessverständnis und ermöglicht durch ein rechnerverarbeitbares Modell, Geschäftsmodelle zu simulieren und fördert somit die Umsetzungsphase für innovative Geschäftsmodelle in der Kreislaufwirtschaft. 


\section{Barrieren und Hemmnisse}

Vergleicht man die Anzahl der existierenden Ansätze, Möglichkeiten und Vorschläge mit den, in der Realität umgesetzten Maßnahmen, so bleibt die Frage offen, warum so wenig in die Realität überführt wird. Diese Frage und die darauf aufbauenden Erklärungsansätze und Lösungsvorschläge werden aktuell viel beforscht (Ritzén und Sandström 2017; Araujo Galvao et al. 2020).

Ritzén und Sandström unterscheiden fünf Gründe (oder Barrieren) für eine erfolgreiche Kreislaufwirtschaft: Finanziell, Strukturell, Betrieblich, Einstellung und Technologisch. Diese Barrieren und welche Vorteile ein MBSE-Ansatz bei der Überwindung dieser Barrieren bietet, stellt Tabelle 1 gegenüber.

Tabelle 1: Gegenüberstellung aktueller Hindernisse in der Kreislaufwirtschaft zum MBSE-Lösungsansatz (* Kategorien von Ritzén und Sandström 2017)

Aktuelle Hindernisse für die Umstellung von der Linearwirtschaft auf eine Kreislaufwirtschaft

\begin{tabular}{lll}
\hline Kategorie* & Begründung* & $\begin{array}{l}\text { Adressierte Lösung durch } \\
\text { MBSE-Ansatz }\end{array}$ \\
\hline Finanziell & Messbarkeit des finanziellen Vorteils & Berechnungsmodell \\
& Finanzielle Rentabilität & - \\
\hline Strukturell & Fehlender Austausch von Informationen & $\begin{array}{l}\text { Informationsaustausch, Visuali- } \\
\text { sierung \& Komplexitätsreduk- } \\
\text { tion }\end{array}$ \\
\hline Operativ & Unklare Verantwortungsverteilung & - \\
\hline Einstellung oder & Infrastruktur und Lieferkettenmanagement \\
Haltung & Risikovermeidung & - \\
\hline Technologisch & Produktdesign & Prädiktion durch Simulation \\
\hline
\end{tabular}

\section{Wertschöpfung und Wertstrommanagement}

Das grundlegende Werkzeug zum Verständnis der Rolle der Technologie für den Wettbewerbsvorteil ist die Wertschöpfungskette. 
Diese Aussage in dem Aufsatz von Michael E. Porter von 1985 ist heute noch immer gültig (Porter 1985). Jede Technologie innerhalb einer Firma leistet einen Betrag zur Wertschöpfung des Gegenstands (ebd.). Die Visualisierung und Bewertung dieser komplexen Vorgänge ermöglicht die Wertstromanalyse. Die Kombination aus graphischen Elementen, einer standardisierten Nomenklatur und dem zugrundeliegenden Ansatz, nicht-wertschöpfende Prozesse weitgehend zu eliminieren, führt zu einer sehr verbreiteten und etablierten Methode in der Praxis - dem Wertstrommanagement.

Die Herausforderung bei der Berechnung und Modellierung von Stoffströmen ist oftmals die fehlende Möglichkeit die Systemgrenze individuell und konsistent zu verändern. Das bedeutet, dass verschiedenen Modellebenen Mikro, Meso und Makroebene konsistent in der Modellierungslogik abgebildet werden und einen Informationsaustausch ermöglichen müssen, um Systemverhalten übergreifend zu analysieren und zu berechnen. Dabei beinhalten die Modellebenen folgende Bestandteile. Die MikroEbene beinhaltet das Produkt und das Material, die Meso-Ebene die Prozessroute und das Unternehmen/Firma und die Makro-Ebene die Region und den Industriebereich die abgewandelte Definition basiert auf dem Ansatz von Kirchherr (Kirchherr et al. 2017).

Die logische Verknüpfung zwischen diesen Ebenen stellt eine Herausforderung in der Modellierung von Systemen dar. Der MBSE-Ansatz und die hier vorgestellte Logik ermöglichen diese Transition zwischen den Ebenen und wird am Beispiel von Materialströmen exemplarisch aufgezeigt.

\section{Wertstromanalyse unter Berücksichtigung von Nachhaltigkeitsaspekten}

Die historische Entwicklung in der Wissenschaft im Kontext der Wertstromanalyse unter Berücksichtigung der Nachhaltigkeit wurde in den letzten 20 Jahren intensiv beforscht und ist auch heute noch ein kontroverses Forschungsfeld. Die systematische Literaturrecherche von Tasdemir und Gazo (2018) gibt einen tiefen Einblick in den Zusammenhang. Der Fokus dieser Recherche lag auf den Forschungsbereichen Lean Manufacturing (LM), Lean Supply Chain Management (LSCM), Nachhaltigkeit und deren Beziehungen zueinander. Die Autoren argumentieren, dass das Konzept der Nachhaltigkeit nicht vermag die Nachhaltigkeit zu messen. Um das zu erreichen müssen andere Methoden mit dem Konzept der Nachhaltigkeit kombiniert werden. Die Recherche ergab, dass die am meiste verwendeten Methode aus dem Bereich des Lean-Managements die folgenden sind: Wertstromanalyse (VSM), 5S, Kaizen, Just-in-Time (JIT), zellulare Fertigung und Single Minute Exchange of Dies (SMED), standardisierte Arbeit und Total Präventive Instandhaltung (TPM) (ebd.). 
Tasdemir und Gazo (2018) leiten aus der Untersuchung eine Vielzahl an Forschungslücken ab. Die in diesem Aufsatz adressierten Forschungslücken sind:

1. Mangel an Bewertungsinstrumenten und Systemen zur Leistungsmessung, um die Effizienz und Nachhaltigkeit von Lieferketten für bestimmte Branchen und Prozesse zu messen,

2. Begrenzte Anwendung von Lean und Nachhaltigkeit in der Lieferkette von KMU,

3. Notwendigkeit, die aktuellen Key Performance Indikatoren (KPIs) neu zu bewerten, um Vergleichbarkeit und Benchmarking über alle Organisationen und Sektoren hinweg zu gewährleisten.

Tabelle 2: Vergleich VSM und Sus-VSM

(angelehnt an Faulkner und Badurdeen 2014; Brown et al. 2014)

\begin{tabular}{|c|c|c|c|c|c|c|}
\hline & & $\begin{array}{l}\text { Konventionelle } \\
\text { Wertstromana- } \\
\text { lyse (VSM) }\end{array}$ & $\begin{array}{l}\text { Erweiterte } \\
\text { Wertstrom- } \\
\text { analyse (Sus- } \\
\text { VSM) }\end{array}$ & $\begin{array}{l}\text { Graphisch } \\
\text { zessschritt }\end{array}$ & Visualisie & rung (pro Pro \\
\hline \multirow{6}{*}{ 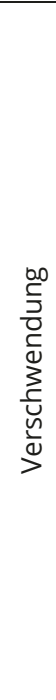 } & \multirow{2}{*}{ Zeit } & \multirow[b]{2}{*}{$X$} & \multirow[b]{2}{*}{$x$} & \multicolumn{2}{|c|}{ xxx Min. } & $\begin{array}{l}\text { Durchlaufzeit }=\mathrm{X} \\
\text { Minuten }\end{array}$ \\
\hline & & & & xxx Min. & xxx Min. & $\begin{array}{l}\text { Bearbeitungs- } \\
\text { zeit }=X\end{array}$ \\
\hline & Material & - & $x$ & \multicolumn{2}{|c|}{ 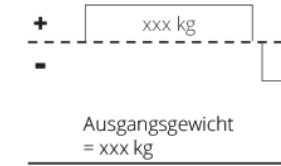 } & $\begin{array}{l}x x x \mathrm{~kg} \\
\begin{array}{l}\text { Finales Gewicht } \\
=x x \times \mathrm{kg}\end{array}\end{array}$ \\
\hline & \multirow{2}{*}{$\begin{array}{l}\text { (Prozess)was- } \\
\text { ser }\end{array}$} & \multirow{2}{*}{ - } & \multirow{2}{*}{$x$} & Benötigt & Verbraucht & Nettoverbrauch \\
\hline & & & & xxx Liter & xxx Liter & xxx Liter \\
\hline & Energie & - & $x$ & $\begin{array}{l}\text { zwischen den } \\
\text { Prozessen }\end{array}$ & $\begin{array}{l}\text { während des } \\
\text { Prozzesses }\end{array}$ & $\begin{array}{l}\text { zwischen den } \\
\text { Prozessen }\end{array}$ \\
\hline \multicolumn{2}{|c|}{$\begin{array}{l}\text { Berufs- oder Arbeits- } \\
\text { platz-risiken (ohne } \\
\text { Lärmschutz \& Ergono- } \\
\text { mie) }\end{array}$} & - & $X$ & \begin{tabular}{l|l}
$: x$ & $H: x$ \\
$: x$ & $S: x$
\end{tabular} & \multicolumn{2}{|c|}{ 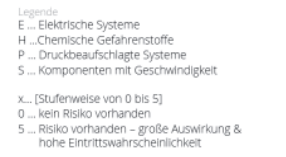 } \\
\hline
\end{tabular}




\section{Der Sus-VSM-Ansatz}

Neben vielen anderen Möglichkeiten Nachhaltigkeitskennzahlen und Produktionsprozesse zu verknüpfen existiert der Ansatz die Wertstromanalyse (engl. Value Stream Mapping - VSM) mit Kennzahlen der Nachhaltigkeit (engl. Sustainability - Sus) zu erweitern. Dieses Konzept wird als Sus-VSM bezeichnet und ist in der aktuellen Forschung ein etabliertes Vorgehen (Faulkner und Badurdeen 2014; Brown et al. 2014). Der Vorteil gegenüber der klassischen VSM besteht darin, dass soziale und ökologische Einflüsse mitberücksichtigt werden können. Die ökologischen Aspekte werden durch die Verbräuche oder Verschwendungen von (Roh-)material, Prozesswasser und Energie berücksichtigt. Die sozialen Aspekte durch die Ergonomie und Berufs- oder Arbeitsplatzrisiken. Die ökonomischen Aspekte werden in Zeitverschwendungen angegeben. Der Vergleich zwischen der konventionellen VSM und der Erweiterung um die Nachhaltigkeitskennzahlen, der Logik und graphischen Darstellung stellt dar. Für die Integration dieser neuen Kennzahlen in eine bestehende Wertstromanalyse werden die Kennzahlen für jeden Prozessschritt ermittelt und graphisch unterhalb oder oberhalb des jeweiligen Prozessschrittes angeordnet.

\section{Methode \& Modell}

Das MBSE-Gesamtmodell wird wie folgt entwickelt. Es erfolgt zunächst eine Kombination der Beschreibung der Wertstromanalyse mit der etablierten grafischen Darstellungsform, um den IST-Zustand der Firma aufzunehmen. Anschließend wird die Logik der Vermeidung von Verschwendung genutzt und unter Berücksichtigung der qualitativen Aussagen der Ökobilanzparameter auf die Stoffströme angewandt.

MBSE- Ansatz für die Stoffstrommodellierung in der Kreislaufwirtschaft

Die vorliegende Studie, die sich im Bereich der Methode für nachhaltigere Produktionsprozesse positioniert (Faulkner und Badurdeen 2014), nutzt die Wertstromanalyse für die Visualisierung und Logik. Für die Kreislaufwirtschafts-spezifischen Merkmale der Prozesse, werden die synthetisierten Merkmale aus dem Literaturreview von Shou (2017) und den Vorschlägen von Helleno (2017) genutzt. In der Gesamtdarstellung wird von Helleno abgewichen und sich an dem Vorgehen von Brown (2014) und Faulkner und Badurdeen (2014) orientiert, mit derselben Begründung wie die Autoren - Übersichtlichkeit. Zusätzlich wird die hier erstmalig beschriebene Erweiterung des Sus-VSMAnsatzes, die für eine bessere Abgrenzung als Sus-VSM+ bezeichnet wird, vorgestellt 
und die Logik anhand einer Case-Study prototypisch mittels SysML im Cameo Systems Modeller implementiert.

\section{Erweiterung des bestehenden Sus-VSM-Ansatzes (Sus-VSM ${ }^{+}$)}

Der Sus-VSM ${ }^{+}$unterscheidet sich gegenüber dem konventionellen Ansatz durch zwei Merkmale. Zum einen durch die Verfeinerung der Materialbeschreibungen für die einzelnen Prozesse und zum anderen durch eine Matching-Logik, die es ermöglicht Materialflüsse quantitativ zu vergleichen und deren Matching-Potenzial zu ermitteln. Der Zusammenhang wird in Form eines neuartigen Koeffizienten - dem Matching-Koeffizient ausgedrückt.

In den Anwendungen des klassichen Sus-VSM Ansatzes ist der Fokus auf der Energieeffizienz, dem Wasserverbrauch und der Materialausnutzung. Bei der Materialausnutzung werden aktuell nur der Rohmaterialbedarf und der Materialausnutzungsgrad berücksichtigt. Diese Perspektive ist zu grob, um mögliche Synergien zwischen Akteuren zu identifizieren. Hierzu müssen folgende materialspezifische Informationen in dem Gesamtmodell zusätzlich erfasst werden:

- Materialart / -klasse

- Materialform

- Gebundener/nicht-gebundener Form (Form, Stoff, Kraftschlüssig, Zusammensetzung: $\mathrm{x} \%$-Anteile)

Werden diese Kriterien für jeden Prozessschritt ermittelt, können daraus folgende Metriken berechnet werden:

- Gesamtbedarf [kg oder Liter]

- Materialausnutzungsgrad / Ausnutzungsgrad

- Anzahl an verschiedenen Stoffen

- Anzahl Input-zu Output-stoffen

Der Vorteil gegenüber der bestehenden Lösung ist, dass diese Werte für jedes verwendete Material ermittelt werden können. Nur durch diese Granularität auf Mikro-Ebene wird es möglich sein, auf Meso- und Makroebene Synergien zu identifizieren.

\section{Partner-Matching Logik}

Die Partnersuche basiert auf der Annahme, dass viele Produktionsabfälle für andere Prozesse als Input genutzt werden können. Die Qualität einer Übereinstimmung (engl. 
Matching) Q_match wird durch die genutzte Stoffmenge (m), den Anteil der verwendeten Stoffmenge im Zielprozess ( $p$ ) (im Verhältnis zum Ausgangsstoff) und der im Stoff gebundenen Energiemenge (E) angegeben.

$$
Q_{\text {match }}=E \times m \times p
$$

mit Stoffanteil

$$
p=\frac{m}{m_{\text {ges }}}=\frac{m}{m_{\text {Ausgangs }}+m}
$$

Er ist das Verhältnis zwischen der Stoffmenge des Ursprungsprozesses $m$ in Bezug zur gesamten Stoffmenge $m_{\text {ges }}$, die sich aus der Summe des Ausgangsstoffes $m_{\text {Ausgangs }}$ und der Stoffmenge des Ursprungsprozesses $m$ ergibt.

Die Voraussetzung für die quantitative Beschreibung einer Übereinstimmung ist, dass Gemeinsamkeiten gefunden werden. Die Schwierigkeit besteht darin, dass Stoffe und deren Merkmale in verschiedenen Industriezweigen stark divergieren und oftmals kleinste Materialeigenschaften darüber entscheiden, ob ein Stoff für einen Prozess geeignet ist. Der MBSE-Ansatz ermöglicht diese Attribute für Industriezweige anzupassen und detaillierter zu beschreiben, ohne die Übersichtlichkeit der Modellierung zu verlieren. Wie der oben beschriebene erweiterte Sus-VSM+ in ein MBSE-Modell überführt werden kann, beschreibt das nächste Kapitel.

\section{Beispielanwendung (Case-Study)}

Der Gesamtprozess wird aus Gründen der Übersichtlichkeit nicht vollständig modelliert. Für die Beispielanwendung wird nur ein Prozessschritt mit kritischen Stoffströmen in dem Modell berücksichtigt. Weiterhin wird eine Beschränkung bei der Potenzialanalyse und anschließenden Auswertung auf die Materialflüsse, um den Fokus nicht zu verlieren, als sinnvoll erachtet. Die aktuell verwendete Auswertungslogik kann den Untersuchungen von Faulkner und Badurdeen und Brown entnommen und auf das hier vorgestellte Beispiel übertragen werden (Faulkner und Badurdeen 2014; Brown et al. 2014). 


\section{Unternehmen, Produkt und Prozessbeschreibung}

Die untersuchte Firma aus Solingen produziert in einem externen Werk neben anderen Produkten Küchenmesser für den europäischen Markt. Diese Küchenmesser bestehen aus einer metallischen Klinge und einem Kunststoffgriff. Der Kunststoffgriff wird bei einer externen Firma durch direktes Anspritzen an den Messerrohling in einem Spritzgussverfahren hergestellt. Der Prozessablauf stellt sich wie folgt dar:

Griffherstellung: An mehreren Arbeitsplätzen produziert jeweils ein Mitarbeiter an einer Spritzgussmaschine aus Messerrohlingen ein vollständiges Messer. Der Mitarbeiter legt Klingenrohlinge in ein Werkzeug. Danach schließt das Werkzeug mechanisch und flüssiger Kunststoff flutet das Werkzeug. Nach wenigen Sekunden öffnet sich das Werkzeug. Die fertigen Messer werden vom Mitarbeiter entnommen und in eine Kiste gelegt, nachdem der Kunststoffgrat vom Messergriff entfernt wurde. Dann legt der Mitarbeiter wieder Messerrohlinge ein - und der Prozess beginnt wieder von vorne.

\section{Implementierung des Sus-VSM+ als MBSE-Modell}

Das Gesamtmodell verknüpft die drei Hauptelemente der Wertstromanalyse. Durch diese Verknüpfung von Produktionsprozess, Materialfluss und Geschäftsprozess, können Kennzahlen für das System ermittelt werden. Die Implementierung des Modells zeigt Abbildung 2. Der linke Bereich bildet die Produktperspektive bestehend aus Produkt, Baugruppen und Bauteilen ab. Die Prozessbeschreibung, bestehend aus Prozessrouten der einzelnen Prozesse, orientiert sich am Wertstromdesign. Diese technische Beschreibung wird um die Geschäftsprozesse ergänzt, um Stückzahlen und Absatzzahlen im Modell berücksichtigen zu können. Das notwendige Material für die Herstellungsprozesse ist in dem hier beschriebenen Ansatz die Verknüpfung zwischen den Perspektiven Prozess und Produkt. Das Produkt durchläuft die Wertschöpfungskette, die aus mehreren Prozessrouten besteht und dadurch das Produkt "wertvoller" macht. Die dafür notwendigen Materialien werden für die Prozessrouten definiert und dann auf Prozessteilebenen verarbeitet. Diese Logik und die daraus ergebene Instanziierung eines Teilprozesses - der Griffherstellung - stellt Abbildung 2 dar.

Wie müssen diese Teilelemente des Gesamtmodells verknüpft werden, um den Herstellungsprozess bei der Griffherstellung abzubilden? Da es aktuell kein standardisiertes Vorgehen zur Prozessmodellierung gibt, wurde ein Framework entwickelt, welches den Fokus auf der Materialausnutzung und der Identifikation der Materialreste in der Prozessroute abbildet (Abbildung 3). Den Prozessrouten sind die Geschäftsprozesse übergeordnet. Die Berücksichtigung der Jahresproduktion ergibt sich aus den Kundenbestellungen und wurde in der Modellierung als Skalierung berücksichtigt. Somit kann 
der Ressourcenverbrauch für den einzelnen Prozessschritt auf den Jahresverbrauch berechnet werden. Der Prozess Griffherstellung wurde durch folgende Modellelemente abgebildet: Griffherstellung, Maschine, Prozess und Randbedingung. Diese Logik verarbeitet einen Materialinputstrom und gibt zwei Stoffströme ab. Dabei wird der eine Stoffstrom an den nachfolgenden Prozessschritt weitergeben (im Modell als Dummy: Nachfolgender Prozessschritt) und der andere Stoffstrom außerhalb der Prozessroute an einen „Container" mit dem Namen Potential: Materialklassen. Dieser Bereich in dem Modell zentralisiert alle anfallenden nicht weiter genutzten Stoffströme der Prozessrouten.

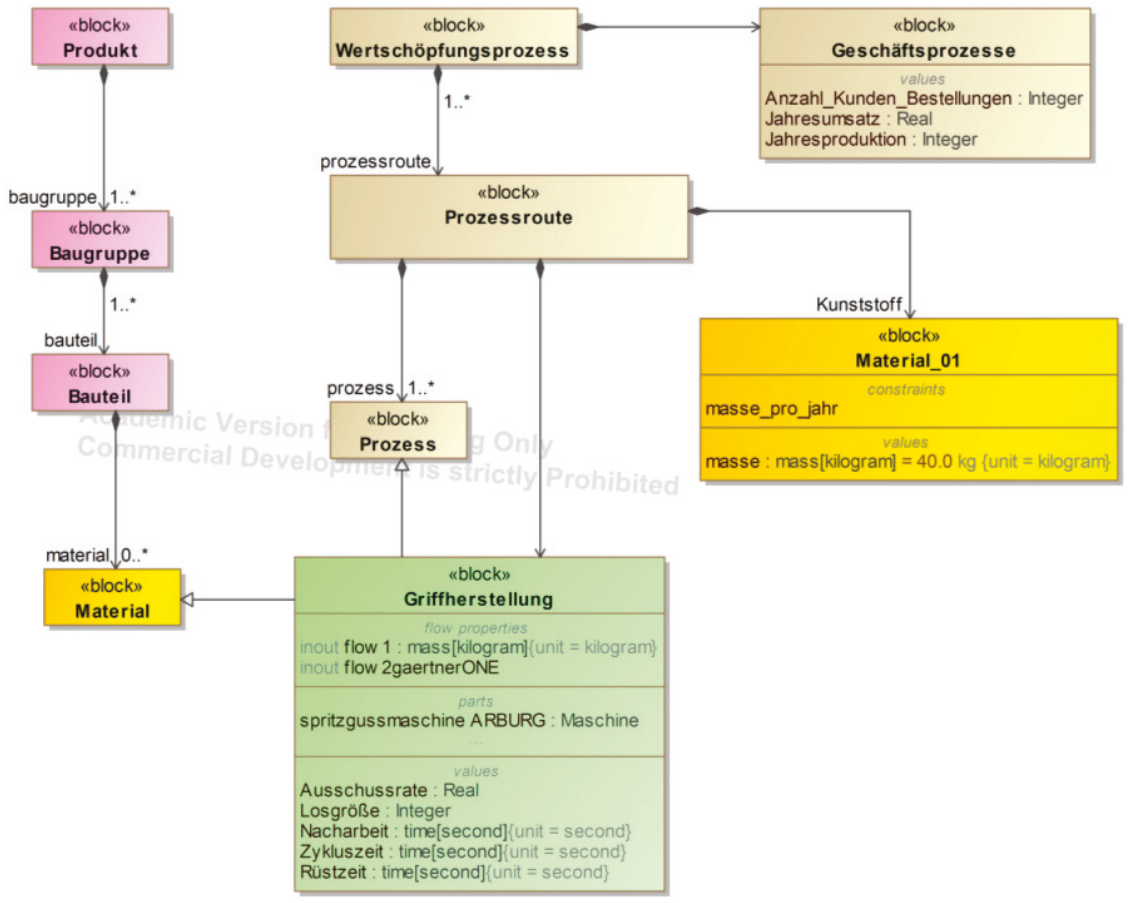

Abbildung 2: MBSE-Gesamtmodell Stoffstrommodellierung

\section{Fazit}

Diese Forschung hat das Ziel, sich der Herausforderungen in der Kreislaufwirtschaft mithilfe von MBSE anzunehmen und Lösungsmöglichkeiten aufzuzeigen. Auf Basis eines etablierten Modellierungsansatzes für Wertstromanalyen unter Berücksichtigung 
von Nachhaltigkeitsaspekten, konnte auf Basis der Herausforderungen der Partnersuche in der Kreislaufwirtschaft, ein erweitertes Modell theoretisch hergeleitet werden. Die exemplarische Implementierung dieser Logik anhand eines Fertigungsprozesses in ein SysML-Modell konnte den Aufbau und die Berechnung validieren.

Durch die Verknüpfung des Inputs, der Gesamtmenge, des Verarbeitungsschritts mit der Materialbeschreibung ist hier erstmalig eine detaillierte Stoffstrommodellierung möglich. Weiterhin lassen sich diese Informationen anforderungsgerecht erweitern, um das Gesamtmodell und die darin enthaltenden Informationen zu verfeinern. Darüber hinaus ist es möglich, Teile des Modells zu extrahieren und externen Partnern zur Verfügung zu stellen, ohne firmeninternes, schützenwertes Wissen zu veröffentlichen. Dadurch können Synergieeffekte durch besseren Informationsaustausch gefördert werden.

Die hier erstmalig beschriebene Logik kann verwendet werden, um die Partnersuche in der Kreislaufwirtschaft signifikant zu verbessern. Dabei helfen die beschriebenen Merkmale und Attribute, die daraus zu berechnenden Kennzahlen und nicht zuletzt die logische Verknüpfung in den gesamten Wertschöpfungsprozess der Unternehmen.

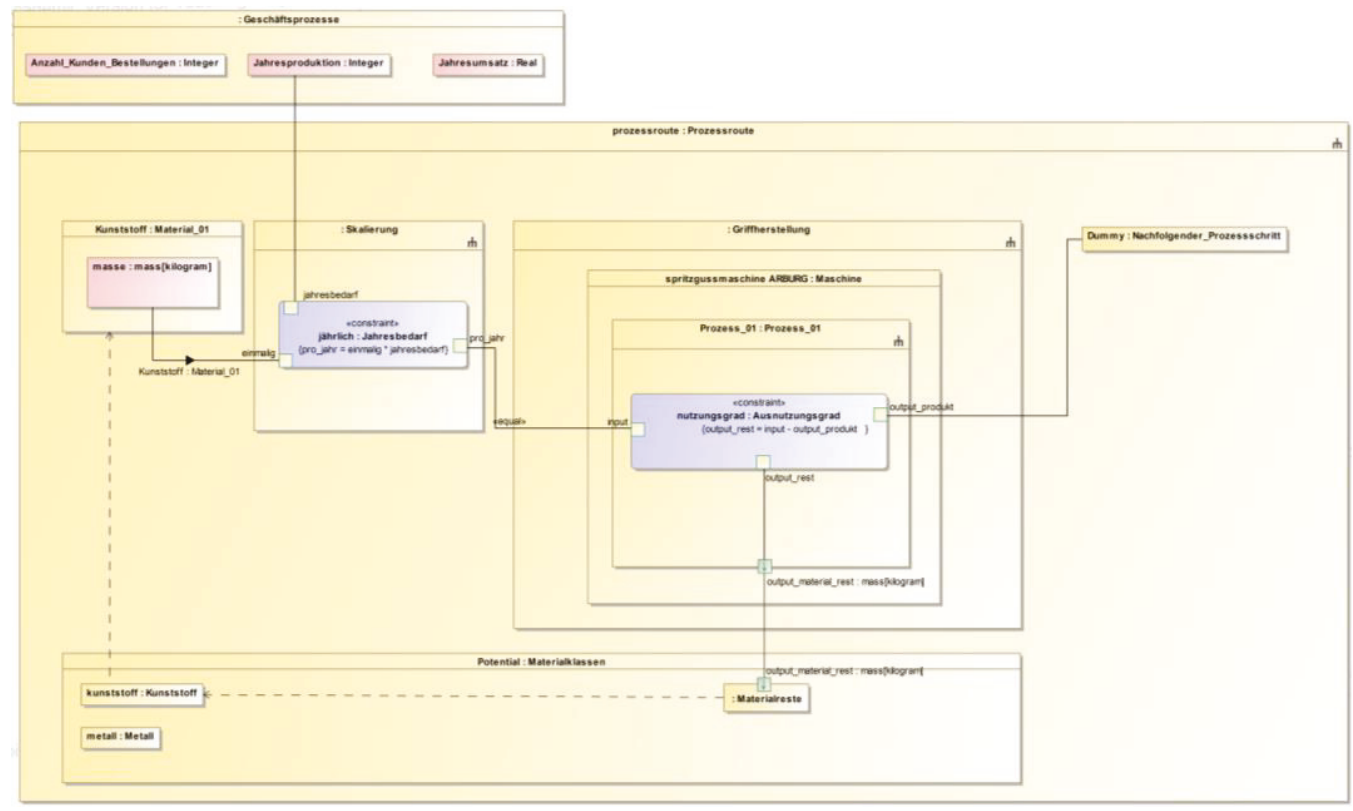

Abbildung 3: MBSE-Modell zur Prozessrouten-Simulation und Stoffstrommodellierung für die Griffherstellung 


\section{Zukünftige Arbeiten}

Die Autoren haben bewusst auf die Erarbeitung von Profilen und übergeordneten Systemengineering Maßnahmen verzichtet; für eine Verbreitung dieses Modells und um die Kompatibilität des Modells zu ermöglichen, sind das notwendige, nächste Schritte.

Darüber hinaus ist es denkbar, dass die Modelle durch detaillierte Sub-Modelle erweitert und verbessert werden. Beispielsweise könnten Maschinenhersteller ihre Prozessdaten zur Verfügung stellen. Für eine nahtlose Integration müssten dafür standardisierte Schnittstellen zum Gesamtmodell definiert werden.

Weiterhin sollten finanzielle, ökologische und soziale Aspekte in Form einer Kennzahl zu berechnen sein. Weiterhin wurden die noch zusätzlich notwendigen Energien und Ressourcen für die Übereinstimmungsbewertung (Matching) aktuell nicht berücksichtigt.

Für eine breite Implementierung und Nutzbarkeit erscheint es mittelfristig sinnvoll, dieses Modell als Basis zu nutzen, um eine webbasierte Plattform für nicht (Sekundär)Ressourcen zu etablieren.

\section{Finanzierung}

Dieses Vorhaben wurde aus Mitteln des Europäischen Fonds für regionale Entwicklung (EFRE) als Teil des Vorhabens mit dem Titel „Regionales Ressourcenmanagement am Beispiel der metallverarbeitenden Industrie im Bergischen Städtedreieck" mit dem Förderkennzeichen EFRE-0400278-454 gefördert.

\section{Literaturverzeichnis}

Andersson, Henric; Herzog, Erik; Johansson, Gert; Johansson, Olof (2010): Experience from Introducing Unified Modeling Language/Systems Modeling Language at Saab Aerosystems. In: Systems Engineering 13 (4), S. 369-380.

Araujo Galvao, Graziela Darla; Homrich, Aline Sacchi; Geissdoerfer, Martin; Evans, Steve; Scoleze Ferrer, Paulo Sergio; Carvalho, Marly M. (2020): Towards a value stream perspective of circular business models. In: Resour. Conserv. Recycl. 162. DOl: 10.1016/j.resconrec.2020.105060.

Barbedienne, Romain; Penas, Olivia; Choley, Jean-Yves; Hehenberger, Peter (2019): Modeling Framework for a Consistent Integration of Geometry Knowledge During Conceptual Design. In: Journal of Computing and Information Science in Engineering 19 (2). DOI: 10.1115/1.4042551.

Benavent Nacher, Sergio; Rosado Castellano, Pedro; Romero Subiron, Fernando; Abellan-Nebot, Jose V. (2020): Multidomain Simulation Model for Analysis of Geometric Variation and Productivity in Multi-Stage Assembly Systems. In: Applied Sciences-Basel 10 (18). DOI: 10.3390/app10186606. 
Bjorkman, Eileen A.; Sarkani, Shahram; Mazzuchi, Thomas A. (2013): Using model-based systems engineering as a framework for improving test and evaluation activities. In: Systems Engineering 16 (3), S. 346-362. D0I: 10.1002/sys.21241.

Bocken, Nancy M. P.; Pauw, Ingrid de; Bakker, Conny; van der Grinten, Bram (2016): Product design and business model strategies for a circular economy. In: Journal of Industrial and Production Engineering 33 (5), S. 308-320. D0l: 10.1080/21681015.2016.1172124.

Bott, Mitch; Mesmer, Bryan (2020): An Analysis of Theories Supporting Agile Scrum and the Use of Scrum in Systems Engineering. In: Engineering Management Journal 32 (2), S. 76-85. D0l: 10.1080/10429247.2019.1659701.

Brown, Adam; Amundson, Joseph; Badurdeen, Fazleena (2014): Sustainable value stream mapping (Sus-VSM) in different manufacturing system configurations: application case studies. In: J. Clean Prod. 85, S. 164-179. DOl: 10.1016/j.jclepro.2014.05.101.

Delbruegger, Tim; Rossmann, Juergen (2019): Representing adaptation options in experimentable digital twins of production systems. In: International Journal of Computer Integrated Manufacturing 32 (4-5), S. 352-365. D0l: 10.1080/0951192X.2019.1599433.

Faulkner, William; Badurdeen, Fazleena (2014): Sustainable Value Stream Mapping (Sus-VSM): methodology to visualize and assess manufacturing sustainability performance. In: J. Clean Prod. 85, S. 8-18. D0I: 10.1016/j.jclepro.2014.05.042.

Global Footprint Network (2020): CALCULATING EARTH OVERSH00T DAY 2020: ESTIMATES POINT TO AUGUST 22ND. Verfügbar unter overshootday.org/content/uploads/2020/06/Earth-Overshoot-Day-2020-Calculation-Research-Report.pdf.

Helleno, Andre Luis; Isaias de Moraes, Aroldo Jose; Simon, Alexandre Tadeu (2017): Integrating sustainability indicators and Lean Manufacturing to assess manufacturing processes: Application case studies in Brazilian industry. In: J. Clean Prod. 153 (1), S. 405-416. DOI: 10.1016/j.jclepro.2016.12.072.

Keskin, Basak; Salman, Baris (2020): Building Information Modeling Implementation Framework for Smart Airport Life Cycle Management. In: Transportation Research Record 2674 (6), S. 98-112. D0I: 10.1177/0361198120917971.

Kirchherr, Julian; Reike, Denise; Hekkert, Marko (2017): Conceptualizing the circular economy: An analysis of 114 definitions. In: Resources, Conservation and Recycling 127, S. 221-232. D0l: 10.1016/j.resconrec.2017.09.005.

Luo, Xiao; Zhang, Lin; Ren, Lei; Lali, Yuanjun (2020): A dynamic and static data based matching method for cloud 3D printing. In: Robotics and Computer-Integrated Manufacturing 61, S. 101858. D0I: 10.1016/j.rcim.2019.101858.

Madni, Azad M. (2015): Expanding Stakeholder Participation in Upfront System Engineering through Storytelling in Virtual Worlds. In: Systems Engineering 18 (1), S. 16-27. D0l: 10.1002/sys.21284.

Mitchell, Steven W. (2014): Transitioning the SWFTS Program Combat System Product Family from Traditional DocumentCentric to Model-Based Systems Engineering. In: Systems Engineering 17 (3), S. 313-329. D0I: 10.1002/sys.21271.

Pieroni, Marina P.P.; McAloone, Tim C.; Pigosso, Daniela C.A. (2019): Business model innovation for circular economy and sustainability: A review of approaches. In: Journal of Cleaner Production 215, S. 198-216. D0l:

10.1016/j.jclepro.2019.01.036.

Poller, Andreas (2020): Exploring and managing the complexity of large infrastructure projects with network theory and model-based systems engineering-The example of radioactive waste disposal. In: Systems Engineering 23 (4), S. 443-459. D0l: 10.1002/sys.21537. 
Porter, Michael E. (1985): TECHNOLOGY AND COMPETITIVE ADVANTAGE. In: Journal of Business Strategy 5 (3), S. 60-78. DOl: 10.1108/eb039075.

Ritzén, Sofia; Sandström, Gunilla Ölundh (2017): Barriers to the Circular Economy - Integration of Perspectives and Domains. In: Procedia CIRP 64, S. 7-12. D0I: 10.1016/j.procir.2017.03.005.

Shou, Wenchi; Wang, Jun; Wu, Peng; Wang, Xiangyu; Chong, Heap-Yih (2017): A cross-sector review on the use of value stream mapping. In: Int. J. Prod. Res. 55 (13), S. 3906-3928. D0l: 10.1080/00207543.2017.1311031.

Sprock, Timothy; Murrenhoff, Anike; McGinnis, Leon F. (2017): A hierarchical approach to warehouse design. In: International Journal of Production Research 55 (21), S. 6331-6343. D0I: 10.1080/00207543.2016.1241447.

Su, Biwei; Heshmati, Almas; Geng, Yong; Yu, Xiaoman (2013): A review of the circular economy in China: moving from rhetoric to implementation. In: Journal of Cleaner Production 42, S. 215-227. DOI: 10.1016/j.jclepro.2012.11.020.

Tasdemir, Cagatay; Gazo, Rado (2018): A Systematic Literature Review for Better Understanding of Lean Driven Sustainability. In: Sustainability 10 (7). DOl: 10.3390/su10072544.

Tchoffa, David; Figay, Nicolas; Ghodous, Parisa; Exposito, Ernesto; Apedome, Kouami Seli; El Mhamedi, Abderrahaman (2019): Dynamic manufacturing network - from flat semantic graphs to composite models. In: International Journal of Production Research 57 (20), S. 6569-6578. D0I: 10.1080/00207543.2019.1570375.

Teece, David J. (2007): Explicating dynamic capabilities: the nature and microfoundations of (sustainable) enterprise performance. In: Strat. Mgmt. J. 28 (13), S. 1319-1350. D0I: 10.1002/smj.640.

Umweltbundesamt (2019): Kunstoffe in der Umwelt. Online verfügbar unter https://bmbf-plastik.de/sites/default/files/2019-06/Umweltbundesamt-2019-Kunststoffe-in-der-Umwelt.pdf, zuletzt geprüft am 20.03.2021.

Wu, Dazhong; Zhang, Linda L.; Jiao, Roger J.; Lu, Roberto F. (2013): SysML-based design chain information modeling for variety management in production reconfiguration. In: Journal of Intelligent Manufacturing 24 (3), S. 575-596. DOI: 10.1007/s10845-011-0585-6.

Yuan, Zengwei; Bi, Jun; Moriguichi, Yuichi (2006): The Circular Economy: A New Development Strategy in China. In: Journal of Industrial Ecology 10 (1-2), S. 4-8. D0I: 10.1162/108819806775545321.

\section{Kontakt}

Franz Wieck

Philipp Kronenberg*

Univ.-Prof. Dr.-Ing. Manuel Löwer

Bergische Universität Wuppertal

Fachgebiet Produktsicherheit und Qualität (PSQ)

*Lehrstuhl für Neue Fertigungstechnologien und Werkstoffe (FUW)

Gaußstraße 20

42119 Wuppertal

www.psq.uni-wuppertal.de 